Proc. Indian Acad. Sci. (Chem. Sci.), Vol. 106, No. 2, April 1994, pp. 433-443.

C) Printed in India.

\title{
Lattice embedding and equilibrium geometry of metal-halogen chains in the two-band extended Hubbard model ${ }^{\dagger}$
}

\author{
Y ANUSOOYA and S RAMASESHA*+ \\ Solid State and Structural Chemistry Unit, Indian Institute of Science, Bangalore 560012, \\ India \\ 'Also at Jawaharlal Nehru Centre for Advanced Scientific Research, Indian Institute of \\ Science Campus, Bangalore 560012, India
}

\begin{abstract}
Two-band extended Hubbard model studies show that the shift in optical gap of the metal-halogen (MX) chain upon embedding in a crystalline environment depends upon alternation in the site-diagonal electron-lattice interaction parameter $\left(\varepsilon_{M}\right)$ and the strength of electron-electron interactions at the metal site $\left(U_{M}\right)$. The equilibrium geometry studies on isolated chains show that the $\mathbf{M X}$ chains tend to distort for alternating $\varepsilon_{\mathrm{M}}$ and small $U_{\mathrm{M}}$ values.
\end{abstract}

Keywords. Two-band extended Hubbard model; lattice embedding; equilibrium geometry; metal-halogen chains.

\section{Introduction}

There has been considerable recent interest in quasi one-dimensional systems containing metal-halogen chains (Tanino and Kobayashi 1983; Fanwick and Huckaby 1987; Yamashita et al 1983; Wada et al 1985; Toriumi et al 1990; Okamato et al 1991). The metal ions are the nickel, palladium or platinum ions and the halide ions are the chloride, bromide or iodide ions. In the crystal, the metal ions are ligated to other ligand molecules and counter ions could also be present between the chains to maintain charge neutrality. A typical system is the $\mathrm{Pt}-\mathrm{Cl}$ system, $\left[\mathrm{Pt}(\mathrm{en})_{2}\right]\left[\mathrm{Pt}(\mathrm{en})_{2} \mathrm{Cl}_{2}\right] \cdot(\mathrm{ClO})_{4}$ (Girlando and Painelli 1991). These systems show an interplay of mixed valence of the metal ion and Peierls' distortion of the one-dimensional $\mathbf{M}-\mathbf{X}$ chain. Thus, there is evidence for strong electron-lattice as well as strong electron-electron interactions in these systems.

The earliest models (Nasu 1984; Bishop and Gammel 1989) for studying the $\mathbf{M}-\mathbf{X}$ systems focussed only on metal ions which contain a single electron in the $d_{z^{2}}$ orbital. The model employed was a half-filled Hubbard model with an effective transfer integral between the transition metal ions (obtained after implicitly integrating out the charge degrees of freedom of the halide ion) and static electron-lattice interaction. The electron-lattice interaction was both site-diagonal leading to different orbital energies at different metal sites as well as site off-diagonal corresponding to an alternation in the intermetal transfer integrals. While this model provided an

\footnotetext{
${ }^{+}$Communication No. 935 from SSCU

* For correspondence
} 
interesting phase diagram in its parameter space, which included phases such as the charge density wave (CDW) and spin density wave (SDW) phases, the model itself was quite unrealistic. Later, theoretical studies (Conradson et al 1988; Gammel et al 1992) employed the two-band extended Hubbard model which explicitly included the orbitals of the halide ion as well. The $p_{z}$ orbital of the halide ion contributed two electrons a one electron was contributed by the $d_{z^{2}}$ orbital of the metal ion. Thus these models are the two-band models at (3/4)ths filling. The electron-lattice interactions included were similar to those in the one-band model. The two-band model studies focussed on structural distortions, electronic spectra and mixed valence in the system and showed that the diverse experimental results were indeed reproduced for different parameter sets of the model. The two-band model studies also have been somewhat incomplete as they do not include the effect of the lattice potential. This could be important since the lattice consists of highly charged metal ions as well as many anions and the Madelung potential associated with the crystal is quite large. Another aspect of the problem that has not been well studied is that of obtaining equilibrium geometry of the $\mathbf{M}-\mathrm{X}$ chains by relaxing the lattice self-consistently. In this paper, we present preliminary results of our studies on the effect of self-consistently embedding small $\mathbf{M}-\mathbf{X}$ chains in the solid, on the optical gap in the system. We also present results of equilibrium geometry calculations of small $\mathbf{M}-\mathrm{X}$ segments by relaxing the bond lengths from a bond order calculation and using Coulson's formula (Coulson 1939). We employ the two-band Pariser-Parr-Pople (PPP) model and carry out complete configuration interaction (CI) calculations in the valence bond (VB) basis. In the next section, we introduce the model Hamiltonian and give some computational details. This is followed by a section on results and discussion. We end this paper with a summary.

\section{Model Hamiltonian and computational details}

The two-band PPP model Hamiltonian is given by

$$
\begin{aligned}
H_{\mathrm{PPP}}= & \sum_{\sigma, i} \varepsilon_{\mathrm{M}} a_{i \sigma}^{*} a_{i \sigma}+\sum_{\sigma, j} \varepsilon_{X} b_{j \sigma}^{*} b_{j \sigma} \\
& +\sum_{i, j, \sigma} t_{i j}\left(a_{i \sigma}^{*} b_{j \sigma}+\text { h.c. }\right) \\
& +\sum_{i} U_{\mathrm{M}} \hat{n}_{i}\left(\hat{n}_{i}-1\right)+\sum_{j} U_{\mathbf{X}} \hat{n}_{j}(\hat{n}-1) \\
& +\sum_{k<1} V_{k l}\left(\hat{n}_{k}-z_{k}\right)\left(\hat{n}_{l}-z_{l}\right),
\end{aligned}
$$

with

$$
\hat{n}_{i}=\sum_{\sigma} a_{i \sigma}^{*} a_{i \sigma} \quad \text { or } \quad \hat{n}_{j}=\sum_{\sigma} b_{j \sigma}^{*} b_{j \sigma}
$$

and

$$
V_{i j}=14.397\left[\left(28 \cdot 794 /\left(U_{i}+U_{j}\right)\right)^{2}+r_{i j}^{2}\right]^{-1 / 2}
$$


where $a_{i \sigma}^{*}\left(a_{i \sigma}\right)$ creates (annihilates) an electron in the metal $d_{z^{2}}$ orbital with spin $\sigma$, similarly the operators $b_{\sigma}^{*}$ and $b_{\sigma}$ are associated with the orbitals of the halide $p_{z}$ orbital. The index $i$, refers to the metal site and $j$, refers to the halogen site. The indices $k$ and $l$ run over all the sites in the chains. The Hubbard on-site repulsion parameters for the metal and the halogen atoms are given by $U_{\mathrm{M}}$ and $U_{\mathrm{X}}$ respectively. The site energy of the metal orbital is given by $\varepsilon_{M}$ and that of the halide ion by $\varepsilon_{X}$. In cases wherein the metal-ion sites are identical, that is when the site-diagonal electron-lattice interaction is identical for all the metal ions, $\varepsilon_{M}$ is taken to be zero. The transfer integral between the metal ion and the halide ion, $t_{0}$, is the same for all bonds in a uniform chain and alternates in a Peierls' distorted chain. In the alternating chain the transfer integral between the $M$ and $X$ ions is taken to be

$$
t=t_{0}+(L-2 \cdot 7035) \times t_{0}
$$

where $L$ is the actual bond length in åmgstroms $(\AA)$ and $t$ and $t_{0}$ are in electronvolts $(\mathrm{eV})$. This choice reflects a linear electron-phonon coupling. The quantities $z_{M}$ and $z_{X}$ fix the correct charges on the ions depending upon the occupancy of the respective orbitals. Thus, when the halide orbital has two, one or zero electrons, the charge on the halide ion would be $-1,0$ or +1 respectively. Similarly, when the metal $d_{z^{2}}$ orbital has two, one or zero electrons, the charge on the metal ion would be 2,3 , or 4 respectively. Thus, the chosen $z_{X}$ and $z_{M}$ values give the lowest energy for the configuration $M^{+4} X^{-1}$ and highest energy for the configuration $M^{+4} X^{+1}$. This is very similar to the parametrization scheme chosen in $\pi$-conjugated systems in the PPP model.

To embed the chain in the solid, we need to evaluate the Madelung potential in the crystal at the metal ions and the halide ion. This is done by using the Ewald procedure (Tosi 1964) wherein the Madelung sum is calculated as a sum of two terms, one involving summation over the direct lattice and another involving summation over the reciprocal lattice. These summations are carried out over a sufficiently large number of neighbours so that the convergence is good to $0.01 \mathrm{eV}$. Since this calculation is the slow step in the entire scheme, we have used the potentials calculated for one structure in the crystal $\left[\mathrm{PdL}_{2}\right]\left[\mathrm{PdBr}_{2} \mathrm{~L}_{2}\right]\left(\mathrm{ClO}_{4}\right)_{4}$ (Yamashita et al 1985) as the potential for all parameters of the model Hamiltonian, (1). The initial charges of the ions used in the calculation corresponds to +3 on the cation and -1 on the anion. To avoid double counting of the interactions, we subtract from the Madelung potential the potential due to ions in the MX chain under consideration. The Madelung potential $V_{M}$ at site $i$ is given by

$$
V_{M}(i)=\sum_{j}^{\prime} z_{j} e^{2} / r_{i j}
$$

The interaction between the Madelung potential and the ions in the chain is now given by the potential $\tilde{V}_{M}(i)$,

$$
\tilde{V}_{M}(i)=V_{M}(i)_{j}-\sum_{E \text { chain }} z_{j} e^{2} / r_{i j}
$$

where $z_{j}$ is the charge on the ion, $j$, and the $r_{i j}$ is the distance between the $j$ th and 
ith ions. In our calculations, we solve the model Hamiltonian for the finite chain exactly and from the ground state eigenfunction obtain the average charges on the metal ions and the halide ion. These charges are then used in the evaluation of the potential due to the lattice and the resulting model Hamiltonian is once again solved. The procedure is repeated iteratively until convergence in the charges on the ions or the energies are within acceptable limits.

To obtain equilibrium geometries in the ground and excited states, we calculate the bond orders of the nearest neighbour bonds in the MX chain. These are given by the expectation value of

$$
\hat{p}_{i j}=\sum_{\sigma} b_{i \sigma}^{*} b_{j \sigma}+b_{j \sigma}^{*} b_{i \sigma}
$$

in the appropriate states. From the bond orders we calculate the relaxed distances between the neighbouring sites using the Coulson (1989) formula,

$$
x=l-(l-s) /\left[l+\left(\left(k_{l} / k_{s}\right) \times(l-p) / p\right)\right],
$$

where $s$ is the bound length of the short bond and $l$ the bond length of the long bond, fixed at 2.496 an $2.911 \AA$, respectively, $p$ is the bond order and $k_{s}$ and $k_{l}$ are the stretching force constants of the short and long bonds taken from literature (Pegiorgi et al 1989) to be 0.73 and 0.61 Newtons per metre. The new bond distances are then used to obtain revised values of the transfer integrals and interaction potentials. The resulting Hamiltonian is then solved and from the eigenstates the bond orders are once again calculated and the whole cycle is repeated until convergence in bond distances or bond orders or the total energies are acceptable.

The model Hamiltonian discussed above conserves total spin. Thus using spin adapted basis functions would greatly reduce the computational effort besides giving the correct spin label to the states. We have employed a VB basis in our calculations. The VB diagrams are easy to construct and manipulate since they can be represented by an integer in the bit representation scheme (Ramasesha and Soos 1984). The VB basis although complete and linearly independent is nonorthogonal and the resulting Hamiltonian matrix is nonsymmetric. However, there exist efficient small matrix algorithms that allow solutions to low-lying states and the lack of symmetry does not lead to any difficulties (Soos and Ramasesha 1990). We have used a cyclic boundary condition in our calculations and the resulting $C_{2}$ symmetry in some cases has also been exploited. Although there exist schemes wherein full spatial symmetry of the Hamiltonian can be exploited within the VB approach, we have not used them in the present studies (Ramasesha and Soos 1993). The Hamiltonian discussed elsewhere in detail (Soos and Ramasesha 1990). The model Hamiltonian spans a finite dimensional Hilbert space and, using the complete and linearly independent VB basis, we obtain an exact representation of the Hamiltonian as a matrix. The eigenstates obtained by solving this matrix are therefore the exact eigenstates of the model Hamiltonian. To obtain the bond orders and charge densities in the desired states, we find it convenient to transform the eigenstates in the VB basis to eigenstates in the Slater determinantal basis. The latter can also be represented as integers in a bit representation scheme and this facilitates the calculation of any desired matrix element (Ramasesha et al 1991). 


\section{Results and discussion}

We have carried out calculations on a chain containing for metal ions and four halide ions. We have calculated the ground and excited state energies, average charge on the ions, energies and charges in the embedded chain, in the ground and dipole allowed excited state. We have also calculated the equilibrium geometry of the chain in the ground state. In the next subsection, we discuss our results of embedding calculations and this will be followed by a subsection on the equilibrium geometry calculations.

\subsection{Effect of embedding on optical gap and mixed valency}

In table 1 is given the variation of the optical gap as a function of the alternation parameter $\delta$, (i) when the site energies at all the metal sites are identical and (ii) when the site energy at a metal site with two short $\mathbf{M}-\mathbf{X}$ bonds is larger than the site energy at a metal site with two long $M-X$ bonds. We find that the effect of embedding

Table 1. The optical gap, in $\mathrm{eV}$, for the cyclic chain of $\mathrm{M}_{4} \mathrm{X}_{4}$ with and without embedding for different alternation parameters $\delta$, for $U_{M}=4 \cdot 0, U_{X}=3.0$ and $\varepsilon_{X}=6.0 \mathrm{eV}$. A refers to uniform $\varepsilon_{\mathrm{M}}=0.0$ and $B$ to $\varepsilon_{M}=0.0$ or $1.0 \mathrm{eV}$ at alternate metal ion sites.

\begin{tabular}{lcccc}
\hline & \multicolumn{3}{c}{ Optical gap } & Shift in \\
\cline { 3 - 4 }$\delta$ & & Before embedding & After embedding & optical gap \\
\hline \multirow{2}{*}{0.10} & A & 1.567 & 1.564 & 0.003 \\
& B & 1.642 & 1.450 & 0.192 \\
0.15 & A & 1.405 & 1.399 & 0.006 \\
& B & 1.793 & 1.589 & 0.204 \\
0.20 & A & 1.246 & 1.236 & 0.010 \\
& B & 1.945 & 1.736 & 0.209 \\
\hline
\end{tabular}

Table 2. Optical gap depencence of $\varepsilon_{\mathrm{X}}$ (both in $\mathrm{eV}$ ) before and after embedding the cyclic chain of $M_{4} X_{4} \cdot \varepsilon_{M}$ has values of 0.0 and $0.5 \mathrm{eV}$ at alternate metal sites. Other model parameters are $U_{M}=4.0 \mathrm{eV}$, $U_{\mathrm{X}}=3.0 \mathrm{eV}$ and $\delta=0.2$.

\begin{tabular}{|c|c|c|c|}
\hline \multirow[b]{2}{*}{$\varepsilon_{X}$} & \multicolumn{2}{|c|}{ Optical gap } & \multirow{2}{*}{$\begin{array}{c}\text { Shift in } \\
\text { optical gat }\end{array}$} \\
\hline & Before embedding & After embedding & \\
\hline$-4 \cdot 0$ & 1.588 & 1.444 & 0.144 \\
\hline-6.0 & 1.517 & 1.360 & 0.157 \\
\hline-8.0 & 1.459 & 1.292 & 0.167 \\
\hline$-10-0$ & 1.411 & 1.236 & 0.175 \\
\hline
\end{tabular}


Table 3. Optical gap (eV) dependence on $U_{M}(\mathrm{eV})$ before and after embedding a cyclic chain of $\mathrm{M}_{4} \mathrm{X}_{4}, \varepsilon_{\mathrm{M}}$ has values $0.0 \mathrm{eV}$ and $1.0 \mathrm{eV}$ at alternate metal sites. $\varepsilon_{\mathrm{X}}=-6 \cdot 0$ and $U_{M}=4 \cdot 0 \mathrm{eV}$.

\begin{tabular}{cccc}
\hline & \multicolumn{2}{c}{ Optical gap } & \\
\cline { 2 - 3 }$U_{\mathbf{X}}$ & Before embedding & After embedding & $\begin{array}{c}\text { Shift in } \\
\text { optical gap }\end{array}$ \\
\hline 1.0 & 1.712 & 1.534 & 0.178 \\
2.0 & 1.815 & 1.621 & 0.194 \\
3.0 & 1.945 & 1.736 & 0.209 \\
4.0 & 2.101 & 1.878 & 0.223 \\
\hline
\end{tabular}

is hardly discernible when the site energies are identical for all alternations. However, when the site energies are different at the metal sites, the optical gap is reduced on embedding. We also find from table 2 that the shift in optical gap is not very sensitive to the site energy at the halide sites or the strength of the electron repulsion $U_{\mathbf{x}}$ (table 3). However, we find that the shift in the optical gap shows an interesting variation with the electron repulsion parameter $U_{\mathrm{M}}$ at the metal site (figure 1). The optical gaps both with and without embedding follow the experimental trend (Aoki et al 1982), namely, the gap decreases with decrease in $U_{x}$ and $\varepsilon_{X}$ which corresponds to changing the halide ion from chloride to bromide to iodide.

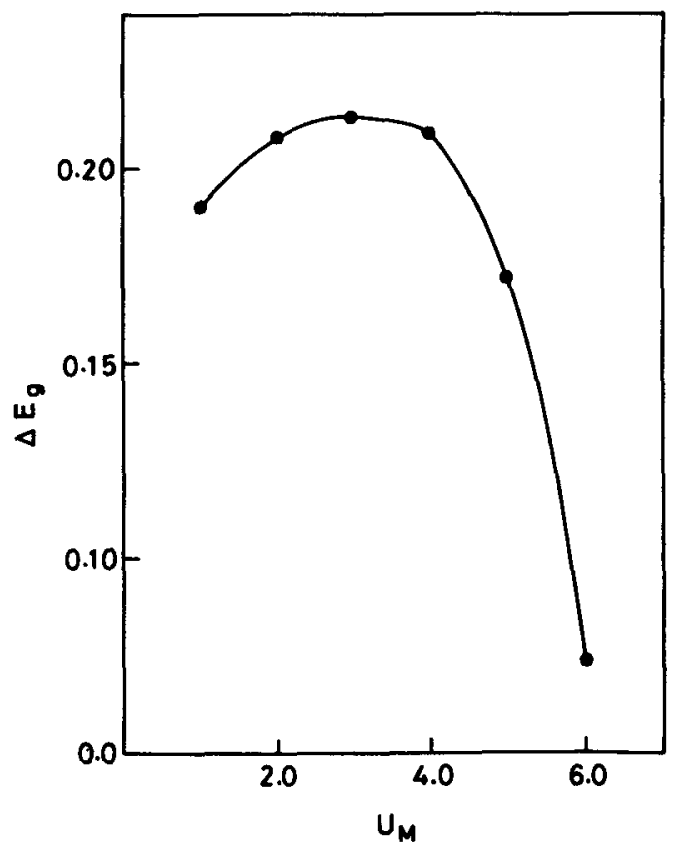

Figure 1. Shift in optical gap upon embedding as a function of the electron repulsion integral $U_{M}$. The alternation $\delta$ is $\mathbf{0 . 2}$ and the site energy at the metal site alternates between 0.0 and $1.0 \mathrm{eV}$. Site energy at the halide site, $\varepsilon_{\mathrm{x}}=-6.0 \mathrm{eV}$ and the halide site electron repulsion integral, $U_{\mathrm{x}}=3.0 \mathrm{eV}$. 


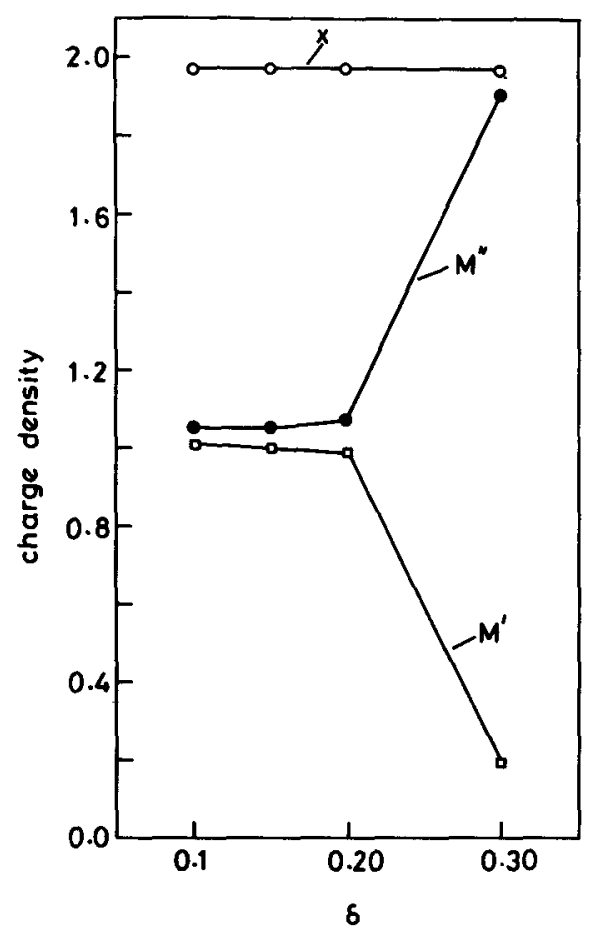

Figure 2. Charge densities in the ground state at the metal sites ( $M$ and $\left.M^{\prime}\right)$ and halogen site $(X)$ as the function of $\delta$ before embedding the cyclic chain of $M_{4} X_{4}$, for $\varepsilon_{X}=-6.0 \mathrm{eV}$, $U_{\mathrm{X}}=3.0 \mathrm{eV}, U_{\mathrm{M}}=4.0 \mathrm{eV}$ and $\epsilon_{\mathrm{M}}=0.0 \mathrm{eV}$ at all metal sites.

The above results show that the important factors affecting the optical gap upon embedding are the site energy differences at the metal sites and the strength of the electron repulsion $U_{M}$ at the metal ion. The degree of mixed valency is sensitive to the site-diagonal distortion. In the absence of site-diagonal distortion, mixed valency is observed only for large values of $\delta$, both before and after embedding (figures 2-3). However, introduction of site-diagonal distortion leads to a large difference in the charge density distribution in the ground state before and after embedding (figure 4 and 5). Thus, the shift in optical gap upon embedding is most sensitive to the site energy differences at the metal ion sites. The shift in optical gap to lower values upon embedding is consistent with the assumption that the dipole allowed state is stabilized to a greater extent, by the Madelung potential, than the ground state because of a higher degree of ionicity in the former state. The interesting dependence of the shift in optical gap with $U_{M}$ is in agreement with the fact that for higher values of $U_{M}$ the degree of mixed valency reduces in the ground and excited states. The fact that embedding does not alter the optical gap significantly when $U_{\mathbf{X}}$ and $\varepsilon_{\mathbf{X}}$ are changed indicates that the halide ion does not directly affect the degree of mixed valency. The charge densities in the ground and the dipole allowed excited states differ in the magnitude of the charge on the metal ions and the halide ion is almost in a closed shell configuration. 


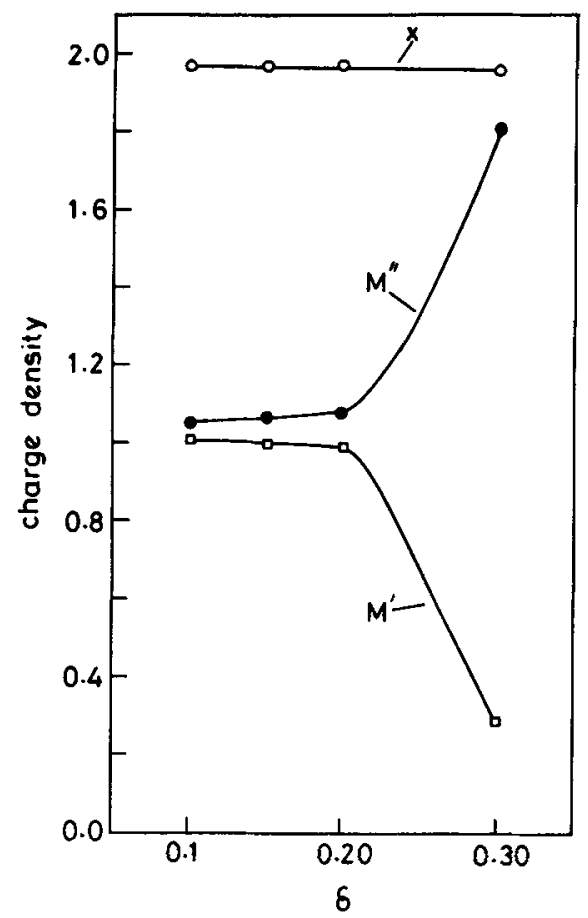

Figure 3. Charge densities in the ground state at the metal sites ( $M$ and $\left.\mathbf{M}^{\prime}\right)$ and the halogen site $(\mathrm{X})$ as the function of $\delta$ after embedding the cyclic chain of $\mathrm{M}_{4} \mathrm{X}_{4}$. All other parameters have the same values as in figure 2.

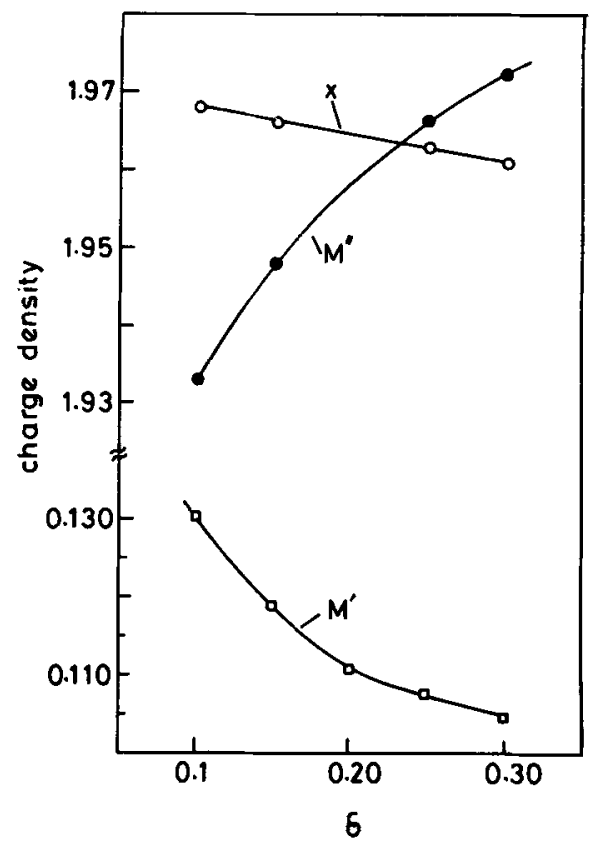

Figure 4. Charge densities in the ground state at the metal sites ( $M$ and $M^{\prime}$ ) and the halogen site $(X)$ as the function of $\delta$ before embedding the cylic chain of $M_{4} X_{4}$, as the funtion of $s$. $\varepsilon_{\mathrm{M}}$ alternates between 1.0 and $0.0 \mathrm{eV}$ at the metal sites and all other parameters are as in figure 2. 


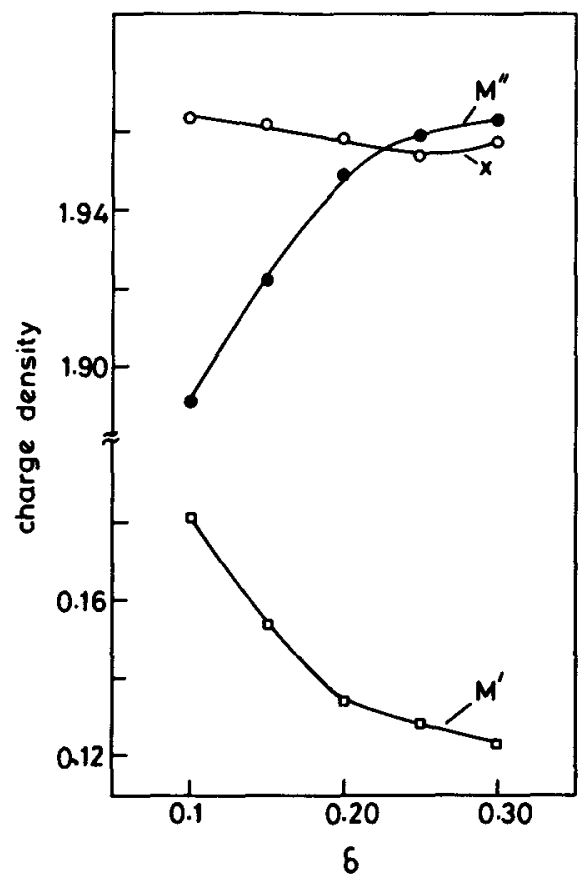

Figure 5. Charge densities in the ground state at metal sites ( $M$ and $\mathbf{M}^{\prime}$ ) and the halogen site $(X)$ as a function of $\delta$, after embedding. All other parameters are as in figure 4.

\subsection{Equilibrium geometry of the $M-X$ chains}

The equilibrium geometry studies have been carried out for many different values of $U_{\mathrm{M}}, U_{\mathrm{X}}, \varepsilon_{\mathrm{M}}$ and $\varepsilon_{\mathrm{X}}$. For large $U_{\mathrm{M}}$ and in the absence of site-diagonal electron-lattice interactions, with a cyclic boundary condition, we do not find evidence for Peierls' distortion of the chain, even when other parameters in the Hamiltonian are varied widely. Even with open boundary condition, there is marginal distortion of the chain as reflected in the slightly unequal equilibrium bond lengths indicating that this is not a finite-size effect. When we introduce site-diagonal electron-lattice interaction by setting different $\varepsilon_{M}$ values at alternate metal ion sites, we find that even with cyclic boundary condition, the equilibrium bond lengths alternate, showing evidence for Peierls' instability (tables 4 and 5). The extent of distortion is insensitive to the site energy (table 4) at the halide ion site as well as to the on-site repulsion parameter (table 5) at the halide site. However, the distortion is sensitive to the Hubbard parameter at the metal ion. At least for the ring of $\mathrm{M}_{4} \mathrm{X}_{4}$, there appears to be a critical value of $U_{M}$ below which the ring distorts even for $\varepsilon_{\mathrm{M}}=0$ at all the sites (tables 4 and 5).

Since the distortion strongly depends upon $\varepsilon_{\mathrm{M}}$, the site-diagonal electron-lattice interaction appears to dictate the structural instability. This parameter is sensitive to the actual halide ion which indeed is in agreement with experiments. If the site energy at the metal-ion site on one sublattice is more negative than at the other, this would favour a higher electron density at those sites. The adjacent halide ions being nearly doubly occupied, such a configuration would not favour delocalization of the 
Table 4. Dependence of equilibrium bond lengths of $M_{4} X_{4}$ cyclic chain on $U_{M}$ and $U_{X}$ when $(A) \varepsilon_{M}=0.0 \mathrm{eV}$ at all metal sites and (B) $\varepsilon_{M}=1.0$ and $0.0 \mathrm{eV}$ at alternate metal sites. $\varepsilon_{\mathrm{X}}=-2.0 \mathrm{eV}$, bond lengths are in angstroms and $U_{\mathrm{M}}$ and $U_{\mathrm{x}}$ are in electron volts.

\begin{tabular}{ccccccc}
\hline & & \multicolumn{3}{c}{ A } & & \multicolumn{2}{c}{ B } \\
\cline { 3 - 4 } \cline { 5 - 6 }$U_{M}$ & $U_{\mathbf{X}}$ & Short bond & Long bond & Short bond & Long bond \\
\hline 2.0 & 1.0 & 2.643 & 2.764 & & 2.629 & 2.778 \\
& 2.0 & 2.651 & 2.756 & & 2.636 & 2.771 \\
& 3.0 & 2.656 & 2.701 & & 2.642 & 2.765 \\
& 4.0 & 2.660 & 2.747 & & 2.647 & 2.760 \\
& 1.0 & 2.704 & 2.704 & & 2.652 & 2.755 \\
& 2.0 & 2.704 & 2.704 & & 2.653 & 2.754 \\
& 3.0 & 2.704 & 2.704 & & 2.654 & 2.753 \\
& 4.0 & 2.704 & 2.704 & & 2.655 & 2.752 \\
\hline
\end{tabular}

Table 5. Dependence of equilibrium bond lengths of $\mathrm{M}_{4} \mathrm{X}_{4}$ cyclic chain on $U_{M}$ and $\varepsilon_{M}$ when $(A) \varepsilon_{M}=0.0 \mathrm{eV}$ at all metal sites and $(\mathrm{B}) \varepsilon_{\mathrm{M}}=1.0$ and $0.0 \mathrm{eV}$ at alternate metal sites. $U_{\mathrm{X}}=1.0 \mathrm{eV}$, bond lengths are in angstroms and $U_{\mathrm{M}}$ and $\varepsilon_{\mathrm{X}}$ are in electron volts.

\begin{tabular}{ccccccc}
\hline & & \multicolumn{3}{c}{ A } & & \multicolumn{2}{c}{ B } \\
\cline { 7 - 7 } \cline { 6 - 7 }$U_{M}$ & $\varepsilon_{\mathbf{X}}$ & Short bond & Long bond & Short bond & Long bond \\
\hline 2.0 & -2.0 & 2.643 & 2.764 & & 2.629 & 2.778 \\
& -4.0 & 2.652 & 2.756 & & 2.637 & 2.770 \\
& -6.0 & 2.659 & 2.748 & & 2.643 & 2.764 \\
& -8.0 & 2.666 & 2.741 & & 2.649 & 2.758 \\
4.0 & -2.0 & 2.704 & 2.704 & & 2.652 & 2.755 \\
& -4.0 & 2.704 & 2.704 & & 2.656 & 2.751 \\
& -6.0 & 2.704 & 2.704 & & 2.660 & 2.747 \\
& -8.0 & 2.704 & 2.704 & & 2.663 & 2.744 \\
\hline
\end{tabular}

electrons. Therefore, the halide ions adjacent to these metal sites would move away. This would result in a stronger $\mathbf{M}-\mathbf{X}$ bond between the halide ion and the metal ion on the other sublattice which has a lower charge density. Such a situation favours greater delocalization of the electrons and hence greater stability. A small $U_{\mathrm{M}}$ also favours distortion of the lattice and this is also in conformity with experiments where the higher row transition elements show a greater tendency for Peierls' distortion. The observation that the Peierls' instability does not depend upon the halide orbital parameters $U_{\mathbf{X}}$ and $\varepsilon_{\mathbf{X}}$ shows that the halide ion only plays an indirect role in the structural distortion in these systems.

To summarize, we have studied the effect of embedding a metal-halogen chain in the crystal on the optical gap in the system. We find that the shift is significant only when the site-diagonal distortion is large. We also find that the shift shows an interesting dependence on the Hubbard parameter at the metal site. The equilibrium 
geometry of the chains also shows a strong dependence on these parameters in conformity with experiments. The above results are based on studies of a cyclic chain of $\mathrm{M}_{4} \mathrm{X}_{4}$ only. To extrapolate to the infinite system we need to carry out similar studies on larger systems such as $M_{6} X_{6}$ and $M_{8} X_{8}$ systems. However, the trends presented above can be justified on physical grounds and we may expect only quantitative differences from further studies.

\section{References}

Aoki R, Hamaue Y, Kida S, Yamashita M, Teramae T, Furuta Y and Kawamori A 1982 Mol. Cryst. Liq. Cryst. 81301

Bishop A R and Gammel T J 1989 Synth. Met. 29 F151

Conradson S D, Stroud M A, Zietlow M H, Swanson B I, Baeriswyl D and Bishop A R 1988 Solid State Commun. 65723

Coulson C A 1939 Proc. R. Soc. London A169 413

Fanwick P E and Huckaby J L 1982 Inorg. Chem. 213067

Gammel J T, Saxena A, Datistic I, Bishop A R and Thillpot S R 1992 Phys. Rev. B45 6408

Girlando A and Painelli A 1991 Synth. Met. 41-43 2721

Nasu K 1984 J. Phys. Soc. Jpn. 53427

Okamoto H, Okaniva K, Mitani T, Toriumi K and Yamashita M 1991 Solid State Commun. 77465

Pegiorgi L, Watcher P, Haruki M and Kurita S 1989 Phys. Rev. B40 3285

Ramasesha S, Albert I D L and Sinha B 1991 Mol. Phys. 72537

Ramasesha S and Soos Z G 1984 J. Chem. Phys. 803278

Ramasesha S and Soos Z G 1993 J. Chem. Phys. 984015

Ramasesha $S$ and Soos Z G in Valence bond theory and chemical structure (eds) D J Klein and N Trinajstic (Amsterdam: Elsevier)

Tanino H and Kobayashi K 1983 J. Phys. Soc. Jpn. 521446

Toriumi K, Okamoto H and Bandow S 1990 Mol. Cryst. Liq. Cryst. 181333

Tosi M P 1964 Solid State Commun. 161

Wada Y, Mitani M and Koda T 1985 J. Phys. Soc. Jpn. 543143

Yamashita M, Toriumi K and Ito T 1983 Inorg. Chem. 221566

Yamashita M, Toriumi K and Ito T 1985 Acta Crystallogr. C41 876 